\title{
SAR Prostate Cancer Disease-Focused Panel report
}

\author{
Silvia D. Chang ${ }^{1} \cdot$ Antonio C. Westphalen ${ }^{2}$ \\ Accepted: 5 November 2020 / Published online: 23 November 2020 \\ (c) Springer Science+Business Media, LLC, part of Springer Nature 2020
}

\section{Mission}

The SAR Prostate Cancer DFP mission is to collaborate with urologists, oncologists, radiation oncologists, and other groups to improve the care of patients with known or suspected prostate cancer through the clinical incorporation of optimized imaging techniques for prostate cancer detection, staging, and treatment.

\section{The structure of the Prostate Cancer DFP}

After the 2020 Society of Abdominal Radiology (SAR) Annual Meeting, the leadership of the Prostate Cancer Disease-Focused Panel (DFP) transitioned from Baris Turkbey and Daniel Margolis to Silvia Chang and Antonio Westphalen.

The Prostate Cancer DFP has 43 members who come from 34 institutions across 4 different countries. Eighteen members have an assigned role, and other eight members are senior advisors. DFP members are identified as full members or early career members. Early career members are those who are junior faculty or in the final year of clinical training. These members are expected to commit at least 3 years to the DFP and after that will be eligible for full membership.

The structure of the Prostate Cancer DFP is being modified to help achieving it is mission. Each subcommittee is now led by two co-chairs, selected based on the results of an internal survey and experience in the field. All members are

Antonio C. Westphalen

acwestph@uw.edu

Silvia D. Chang

silvia.chang@vch.ca

1 Department of Radiology, Vancouver General Hospital, University of British Columbia, 899 West 12th Avenue, Vancouver, BC V5Z 1M9, Canada

2 Department of Radiology and Radiation Oncology, University of Washington, 1959 NE Pacific St, BB308, Seattle, WA 98195, USA allowed to officially participate in one or two subcommittees, but all are welcome and encouraged to collaborate with any projects brought-forth by any subcommittee.

The mission and goals of the Education and Clinical Practice subcommittees have been revised. The Education Subcommittee has been tasked with helping to organize the SAR Annual Meeting Prostate Cancer Hands-on Workshop and lecture series, the DFP teaching file and journal club, as well as oversee the Prostate Cancer DFP mentorship program, all aligned to and in collaboration with other ongoing SAR efforts. Dr. Purysko and Dr. Sai co-chair the subcommittee. Dr. Arora will co-direct and later lead the organization of the hands-on prostate MRI workshop. The Clinical Practice Subcommittee is responsible for the review and assessment of current practice guidelines. Its major goals are to identify opportunities to expand the role of imaging in the care of men with prostate cancer and participate in efforts to develop documents/statements based on the best evidence to support the use of imaging. Dr. Yu and Dr. Gupta co-chair the subcommittee.

The Clinical Trials subcommittee was dissolved and replaced by the Research and AI Subcommittee. Its tasks are to develop Prostate Cancer DFP research initiatives and promote collaborations with other DFPs, groups, and institutions. Dr. Turkbey and Dr. Haider co-chair the subcommittee.

The Social Media and Website Subcommittees were combined into the new Engagement, Social Media, and Website Subcommittee. Its members are responsible for the planning of social activities, supervision of the DFP social media accounts, website maintenance and content distribution, expansion of our network, and Prostate Cancer DFP marketing. These activities will be carried out in conjunction with the SAR Oversight Committee and Veritas. Dr. Ghafoor chairs the subcommittee, and the selection of a co-chair is under way.

Two new subcommittees have been created: the Diversity and the Outreach Subcommittees. The Diversity Subcommittee will promote inclusion and diversity within the Prostate Cancer DFP and in clinical practice, identify disparities 
in diagnosis of prostate cancer, and develop/collaborate with efforts to minimize these disparities. Dr. Lall and Dr. Margolis have agreed to co-chair the subcommittee. The Outreach Subcommittee tasks are to foster and maintain relationships and partnerships with members of the community and other institutions/organizations, as well as identify potential new Prostate Cancer DFP members. The subcommittee is cochaired by Dr. Patel and Dr. Kim.

In addition to our subcommittees, the Prostate Cancer DFP has liaison members to several national and international societies (Asian Society of Abdominal Radiology, European Society of Urogenital Radiology, American College of Radiology, Society of Interventional Radiology, Society of Nuclear Medicine and Molecular Imaging, and the American Urological Association) and a Consultant Urologist, Dr. Leonard Marks.

The DFP is currently organizing a mentoring program which will leverage on the experience of the senior members, as well as on the experience of other panels and the SAR mentoring program, to promote, and support junior colleagues and trainees who have a passion for prostate cancer.

\section{Accomplishments of the past year}

\section{Cases of the week}

Thirty-five new cases were added to the database since 06/01/2019. A total of 167 cases are publicly available in the website (https://www.abdominalradiology.org/page/DFPpr ostateTC/Prostate-Cancer-DFP-Teaching-Case-of-theWeek.htm), and to improve access all have been classified by theme, level of difficulty, and Prostate Imaging Reporting \& Data System (PI-RADS) category. There are also links to the AUA-SAR Joint Consensus Statement on Prostate MRI, and other useful online and journal resources.

The recently published Special Section on Male Pelvis (https://link.springer.com/journal/261/45/7) and this Special Issue of Abdominal Radiology highlight the work of a number of panelists. The DFP also published an assessment of the variability of PI-RADS across 26 institutions (https ://doi.org/10.1148/radiol.2020190646) which has received a fair amount of attention, in particular in the various social media platforms. It is expected that the results of this study will lead to changes in PI-RADS and will stimulate further efforts to improve and evaluate the quality of prostate magnetic resonance imaging (MRI). A survey by this panel on the practice patterns and challenges of performing and interpreting prostate MRI has been accepted for publication in AJR. The information gathered from this survey will help design further education programs and guide further refinements to the next version of PI-RADS. In addition, the DFP panelists have published many other manuscripts in collaborations that involve also colleagues from outside the DFP and other specialties.

Members also continue to be very active on social media, with hundreds of tweets on Twitter and other platforms. Very recently we have passed the threshold of 1000 (1146 as of September 2020) followers.

One of our most popular offerings has been the SAR Annual Meeting Hands-on Prostate MRI Workshop, held in combination with the prostate track at the Annual Meeting. The most recent workshop was runaway success again, with 45 attendees, a good number considering that the workshop coincided with early part of the coronavirus pandemic. The DFP is committed to give an opportunity to younger colleagues to participate in workshop or as speaker in the prostate track. As in the previous 3 or 4 years, approximately half of the collaborators rotate out and new people are paired with more experienced members. We have also used the workshop to expand the reach of the SAR and DFP beyond North America, by inviting colleagues from other continents to participate as moderators.

The Clinical Practice Subcommittee, led by Steve Eberhardt and Nelly Tan have produce podcasts in which the value and applications of PSMA and fluciclovine PET are discussed, and PI-RADS v2.1 explained. In addition, as part of their directive to improve the value to practicing radiologists, the subcommittee began a collaboration with www. Radiologylnfo.org on new and updated articles for patients and colleagues.

Inter-societal collaborations have been one of the strengths of our DFP and our panelists continue to work on established working groups with ASTRO and SIR to develop guidelines and educational collaborations. The recently published Optimum Imaging Strategies for Advanced Prostate Cancer: ASCO Guideline (J Clin Oncol. 2020 Jun 10;38(17):1963-1996) is an example of these collaborations.

Very recently Dr. Bathala led a virtual symposium with experts of various specialties to discuss imaging diagnosis, histological assessment, treatment, and outcomes of extraprostatic extension of cancer. The event was a huge success with more than 150 attendees from around the globe.

The DFP has twice held the Prostate DFP Café, a 1-h virtual social gathering where members are welcome to discuss any topics. These are unstructured network events aimed at fostering new friendships, development of new collaborations, and discussion of ideas.

Dr. Surasi is collecting survey data to determine the impact COVID had on imaging assessment of men with suspected or known prostate cancer across the nation. It is expected that these data will allow the DFP to determine the potential immediate future needs of men who have had delayed management and propose potential solutions to mitigate any negative impact of the pandemic. 


\section{Other planned or ongoing activities/projects}

The Prostate Cancer DFP, along with its oversight committee, is seeking to expand its collaborations with members of other institutions, including the National Comprehensive Cancer Network and the RSNA, possibly through a partnership with the RSNA Case Collection to expand the reach of our Case of the Week database. The DFP would like to explore a partnership with the AdMeTech Foundation. The “AdMeTech Foundation's priority focus is on ending the prostate cancer crisis." The foundation Awareness, Education, and Advocacy Programs and its research program are aligned with our own goals. Several panelists participate or participated in its Annual Global Summit on Precision Diagnosis and Treatment for Prostate Cancer, a potential venue for fostering new and strengthening collaborations with colleagues from various other institutions and specialties.

In addition to the Podcasts, the Clinical Practice Subcommittee considers developing vodcasts as well, i.e., video presentations with educational material/discussions for practicing radiologists, clinicians, and patients.
The DFP would like to collaborate with the ACR to develop modifications to the current PI-RADS guidelines and develop new ones, e.g., for post-treatment assessment. Similarly, the DFP would like to join forces with the SIR and plan a focal therapy workshop.

This is a summary of our most recent activities. The SAR Prostate Cancer DFP has been and will continue to be very successful in its endeavors. And this is because all members share these same traits: camaraderie, professionalism, and passion for our field.

Thank you, keep safe, and keep active!

Publisher's Note Springer Nature remains neutral with regard to jurisdictional claims in published maps and institutional affiliations. 